\title{
Positive Solutions for the Initial Value Problem of Fractional Evolution Equations
}

\author{
He Yang ${ }^{1}$ and Yue Liang ${ }^{2}$ \\ ${ }^{1}$ Department of Mathematics, Northwest Normal University, Lanzhou 730070, China \\ ${ }^{2}$ Science College, Gansu Agricultural University, Lanzhou 730070, China \\ Correspondence should be addressed to He Yang; yanghe256@163.com
}

Received 9 December 2012; Accepted 19 February 2013

Academic Editor: Changbum Chun

Copyright (c) $2013 \mathrm{H}$. Yang and Y. Liang. This is an open access article distributed under the Creative Commons Attribution License, which permits unrestricted use, distribution, and reproduction in any medium, provided the original work is properly cited.

By using the fixed point theorems and the theory of analytic semigroup, we investigate the existence of positive mild solutions to the Cauchy problem of Caputo fractional evolution equations in Banach spaces. Some existence theorems are obtained under the case that the analytic semigroup is compact and noncompact, respectively. As an example, we study the partial differential equation of the parabolic type of fractional order.

\section{Introduction}

The differential equations involving fractional derivatives in time have recently been studied extensively. One can see, for instance, the monographs [1-5] and the survey [6-8]. In particular, there has been a significant development in fractional evolution equations. Existence of solutions for fractional evolution equations has been studied by many authors during recent years. Many excellent results are obtained in this field; see [9-19] and the references therein. In [9, 10], El-Borai first constructed the type of mild solutions to fractional evolution equations in terms of a probability density. And then the author investigated the existence, uniqueness, and regularity of solutions of fractional integrodifferential equations in [11, 12]. Recently, this theory was developed by Zhou et al. [13-16]. Particularly, they studied the existence and controllability of mild solution of fractional delay integrodifferential equations with a compact analytic semigroup in [16]. In [17-19], the authors studied the existence of mild solutions of fractional impulsive delay or impulsive evolution equations. But as far as we know, there are no results on the existence of positive solutions of fractional evolution equations.

In this paper, by using the fixed point theorems combined with the theory of analytic semigroup, we investigate the existence of positive mild solutions for the initial value problem (IVP) of fractional evolution equations in Banach space $X$ as

$$
\begin{gathered}
D_{0}^{q} u(t)+A u(t)=f(t, u(t)), \quad t>0, \\
u(0)=u_{0},
\end{gathered}
$$

where $D_{0}^{q}$ denotes the Caputo fractional derivative of order $q \in(0,1)$ with the lower limits zero, $-A: D(A) \subset X \rightarrow$ $X$ is the infinitesimal generator of an analytic semigroup $S(t)(t \geq 0)$ of uniformly bounded linear operators, and $f$ is the nonlinear term and will be specified later.

The rest of this paper is organized as follows. In Section 2, some preliminaries are given on the fractional power of the generator of the analytic semigroup and the definition of mild solutions of IVP(1). In Section 3, we study the existence of positive mild solutions for the IVP(1). In Section 4, an example is given to illustrate the applicability of abstract results obtained in Section 3.

\section{Preliminaries}

In this section, we introduce some basic facts about the fractional power of the generator of analytic semigroup and the fractional calculus that are used throughout this paper. 
Let $X$ be a Banach space with norm $\|\cdot\|$. Throughout this paper, we assume that $-A: D(A) \subset X \rightarrow X$ is the infinitesimal generator of an analytic semigroup $S(t)(t \geq 0)$ of uniformly bounded linear operator in $X$; that is, there exists $M \geq 1$ such that $\|S(t)\| \leq M$ for all $t \geq 0$. Without loss of generality, let $0 \in \rho(-A)$, where $\rho(-A)$ is the resolvent set of $-A$. Then for any $\alpha>0$, we can define $A^{-\alpha}$ by

$$
A^{-\alpha}:=\frac{1}{\Gamma(\alpha)} \int_{0}^{\infty} t^{\alpha-1} S(t) d t
$$

Then $A^{\alpha}$ can be defined by $A^{\alpha}:=\left(A^{-\alpha}\right)^{-1}$ because $A^{-\alpha}$ is one to one. It can be shown that each $A^{\alpha}$ has dense domain and that $D\left(A^{\beta}\right) \subset D\left(A^{\alpha}\right)$ for $0 \leq \alpha \leq \beta$. Moreover, $A^{\alpha+\beta} x=$ $A^{\alpha} A^{\beta} x=A^{\beta} A^{\alpha} x$ for every $\alpha, \beta \in \mathbb{R}$ and $x \in D\left(A^{\mu}\right)$ with $\mu:=\max \{\alpha, \beta, \alpha+\beta\}$, where $A^{0}=I, I$ is the identity in $X$ (for proofs of these facts we refer to the literature [20-22]).

We denote by $X_{\alpha}$ the Banach space of $D\left(A^{\alpha}\right)$ equipped with norm $\|x\|_{\alpha}=\left\|A^{\alpha} x\right\|$ for $x \in D\left(A^{\alpha}\right)$, which is equivalent to the graph norm of $A^{\alpha}$. Then we have $X_{\beta} \hookrightarrow X_{\alpha}$ for $0 \leq$ $\alpha \leq \beta \leq 1$ (with $X_{0}=X$ ), and the embedding is continuous. Moreover, $A^{\alpha}$ has the following basic properties.

Lemma 1 (see [23]). $A^{\alpha}$ has the following properties.

(i) $S(t): X \rightarrow X_{\alpha}$ for each $t>0$ and $\alpha \geq 0$.

(ii) $A^{\alpha} S(t) x=S(t) A^{\alpha} x$ for each $x \in D\left(A^{\alpha}\right)$ and $t \geq 0$.

(iii) For everyt $>0, A^{\alpha} S(t)$ is bounded in $X$ and there exists $M_{\alpha}>0$ such that

$$
\left\|A^{\alpha} S(t)\right\| \leq M_{\alpha} t^{-\alpha}
$$

Let $J$ be a closed interval on $\mathbb{R}^{+}=[0, \infty)$. In the following we denote by $C\left(J, X_{\alpha}\right)$ the Banach space of all continuous functions from $J$ into $X_{\alpha}$ endowed with supnorm given by $\|u\|_{C}=\sup _{t \in J}\|u(t)\|_{\alpha}$ for $u \in C\left(J, X_{\alpha}\right)$. For any $t \geq 0$, denote by $S_{\alpha}(t)$ the restriction of $S(t)$ to $X_{\alpha}$. From Lemma 1(i) and (ii), for any $x \in X_{\alpha}$, we have

$$
\begin{aligned}
\|S(t) x\|_{\alpha} & =\left\|A^{\alpha} \cdot S(t) x\right\|=\left\|S(t) \cdot A^{\alpha} x\right\| \\
& \leq\|S(t)\| \cdot\left\|A^{\alpha} x\right\|=\|S(t)\| \cdot\|x\|_{\alpha}, \\
\|S(t) x-x\|_{\alpha} & =\left\|A^{\alpha} \cdot S(t) x-A^{\alpha} x\right\| \\
& =\left\|S(t) \cdot A^{\alpha} x-A^{\alpha} x\right\| \longrightarrow 0
\end{aligned}
$$

as $t \rightarrow 0$. Therefore, $S(t)(t \geq 0)$ is a strongly continuous semigroup in $X_{\alpha}$, and $\left\|S_{\alpha}(t)\right\|_{\alpha} \leq\|S(t)\|$ for all $t \geq 0$. To prove our main results, the following lemma is also needed.

Lemma 2 (see [24]). If $S(t)(t \geq 0)$ is a compact semigroup in $X$, then $S_{\alpha}(t)(t \geq 0)$ is a compact semigroup in $X_{\alpha}$, and hence it is norm continuous.

Let us recall the following known definitions in fractional calculus. For more details, see $[9,13-16,18,19]$.
Definition 3. The fractional integral of order $\sigma>0$ with the lower limits zero for a function $f$ is defined by

$$
I_{0}^{\sigma} f(t)=\frac{1}{\Gamma(\sigma)} \int_{0}^{t}(t-s)^{\sigma-1} f(s) d s, \quad t>0,
$$

where $\Gamma$ is the gamma function.

The Riemann-Liouville fractional derivative of order $n-$ $1<\sigma<n$ with the lower limits zero for a function $f$ can be written as

$$
\begin{array}{r}
{ }^{L} D_{0}^{\sigma} f(t)=\frac{1}{\Gamma(n-\sigma)} \frac{d^{n}}{d t^{n}} \int_{0}^{t}(t-s)^{n-\sigma-1} f(s) d s, \\
t>0, n \in \mathbb{N} .
\end{array}
$$

Also the Caputo fractional derivative of order $n-1<\sigma<n$ with the lower limits zero for a function $f \in C^{n}[0, \infty)$ can be written as

$$
\begin{array}{r}
D_{0}^{\sigma} f(t)=\frac{1}{\Gamma(n-\sigma)} \int_{0}^{t}(t-s)^{n-\sigma-1} f^{(n)}(s) d s, \\
t>0, n \in \mathbb{N} .
\end{array}
$$

Remark 4. (1) The Caputo derivative of a constant is equal to zero.

(2) If $f$ is an abstract function with values in $X$, then integrals which appear in Definition 3 are taken in Bochner's sense.

Lemma 5 (see [14]). A measurable function $h: J \rightarrow X$ is Bochner integrable if $\|h\|$ is Lebesgue integrable.

For $x \in X$, we define two families $\{U(t)\}_{t \geq 0}$ and $\{V(t)\}_{t \geq 0}$ of operators by

$$
\begin{gathered}
U(t) x=\int_{0}^{\infty} \eta_{q}(\theta) S\left(t^{q} \theta\right) x d \theta, \\
V(t) x=q \int_{0}^{\infty} \theta \eta_{q}(\theta) S\left(t^{q} \theta\right) x d \theta, \quad 0<q<1,
\end{gathered}
$$

where

$$
\eta_{q}(\theta)=\frac{1}{q} \theta^{-1-1 / q} \rho_{q}\left(\theta^{-1 / q}\right)
$$

$$
\begin{aligned}
& \rho_{q}(\theta) \\
& =\frac{1}{\pi} \sum_{n=1}^{\infty}(-1)^{n-1} \theta^{-q n-1} \frac{\Gamma(n q+1)}{n !} \sin (n \pi q), \quad \theta \in(0, \infty),
\end{aligned}
$$

where $\eta_{q}$ is a probability density function defined on $(0, \infty)$, which has properties $\eta_{q}(\theta) \geq 0$ for all $\theta \in(0, \infty)$ and $\int_{0}^{\infty} \eta_{q}(\theta) d \theta=1$. It is not difficult to verify (see [14]) that for $\mu \in[0,1]$, we have

$$
\int_{0}^{\infty} \theta^{\mu} \eta_{q}(\theta) d \theta=\frac{\Gamma(1+\mu)}{\Gamma(1+q \mu)}
$$


Clearly, if the semigroup $S(t)(t \geq 0)$ is positive, then, by the definitions, the operators $U(t)$ and $V(t)$ are also positive for all $t \geq 0$.

The following lemma follows from the results in $[14$, Lemma 2.9] and [15, Lemmas 3.2-3.5].

Lemma 6. The operators $U(t)$ and $V(t)$ have the following properties.

(i) For any fixed $t \geq 0$ and any $x \in X_{\alpha}$, one has

$$
\begin{gathered}
\|U(t) x\|_{\alpha} \leq M\|x\|_{\alpha}, \\
\|V(t) x\|_{\alpha} \leq \frac{q M}{\Gamma(1+q)}\|x\|_{\alpha}=\frac{M}{\Gamma(q)}\|x\|_{\alpha} .
\end{gathered}
$$

(ii) The operators $U(t)$ and $V(t)$ are strongly continuous for all $t \geq 0$.

(iii) If the semigroup $S(t)(t \geq 0)$ is compact, then $U(t)$ and $V(t)$ are compact operators in $X$ for $t>0$.

(iv) If the semigroup $S_{\alpha}(t)(t \geq 0)$ is norm continuous, then the restriction of $U(t)$ to $X_{\alpha}$ and the restriction of $V(t)$ to $X_{\alpha}$ are uniformly continuous for $t>0$.

Definition 7 (see $[25,26]$ ). Let $B$ be a bounded set of a real Banach space $E$. Set $\beta(B)=\inf \{\delta>0: B$ can be expressed as the union of a finite number of sets such that the diameter of each set does not exceed $\delta$; that is, $B=\cup_{i=1}^{m} B_{i}$ with $\operatorname{diam}\left(B_{i}\right) \leq$ $\delta, i=1,2, \ldots, m\} . \beta(B)$ is called the Kuratowski measure of noncompactness of set $B$.

It is clear that $0 \leq \beta(B)<\infty$. For the Kuratowski measure of noncompactness, we have the following well-known results.

Lemma 8 (see [26]). If $D \in C(J, E)$ is bounded and equicontinuous, then

$$
\beta(D)=\beta(D(J))=\max _{t \in J} \beta(D(t)),
$$

where $D(J)=\{x(t): x \in D, t \in J\}$.

Lemma 9 (see [27]). Let $D$ be a countable set of strongly measurable function $x: J \rightarrow E$ such that there exists an $M \in L\left(J, \mathbb{R}^{+}\right)$such that $\|x(t)\| \leq M(t)$ a.e., $t \in J$ for all $x \in D$. Then $\beta(D(t)) \in L\left(J, \mathbb{R}^{+}\right)$and

$$
\beta\left(\left\{\int_{J} x(t) d t: x \in D\right\}\right) \leq 2 \int_{J} \beta(D(t)) d t .
$$

Lemma 10 (see [25] Mönch fixed point theorem). Let $B$ be a closed and convex subset of $E$ and $y_{0} \in B$. Assume that the continuous operator $A: B \rightarrow B$ has the following property: $D \subset B$ is countable, and $D \subset \overline{\mathrm{Co}}\left(\left\{y_{0}\right\} \cup A(D)\right) \rightarrow D$ is relatively compact. Then $A$ has a fixed point in $B$.

Based on an overall observation of the previous related literature, in this paper we adopt the following definition of mild solution of IVP(1).
Definition 11. By a mild solution of the IVP(1), one means a function $u \in C\left(J, X_{\alpha}\right)$ satisfying

$$
u(t)=U(t) u_{0}+\int_{0}^{t}(t-s)^{q-1} V(t-s) f(s, u(s)) d s
$$

for all $t \in J$.

\section{Existence of Positive Mild Solutions}

In this section, we introduce the existence theorems of positive mild solutions of the IVP(1). The discussions are based on fractional calculus and fixed point theorems.

Let $\lambda_{1}$ be the smallest positive real eigenvalue of the linear operator $A$, and let $e_{1} \in D(A)$ be the positive eigenvector corresponding to $\lambda_{1}$. For any $T>0$ and $r>0$, we write

$\Omega_{r}$

$$
:=\left\{u \in C\left([0, T], X_{\alpha}\right):\|u(t)\|_{\alpha} \leq r, u(t) \geq \sigma e_{1}, t \in[0, T]\right\},
$$

where $\sigma>0$ is a constant. Our main results are as follows.

Theorem 12. Let $-A: D(A) \subset X \rightarrow X$ be the infinitesimal generator of a positive and compact analytic semigroup $S(t)(t \geq 0)$ of uniformly bounded linear operators. Assume that $f \in C\left(\mathbb{R}^{+} \times X_{\alpha}, X\right)$ satisfies the following conditions.

$\left(H_{1}\right)$ For any $u \in \Omega_{r}$, one has

$$
f(t, u(t)) \geq f\left(t, \sigma e_{1}\right) \geq \lambda_{1} \sigma e_{1}, \quad t \in[0, T] .
$$

$\left(\mathrm{H}_{2}\right) f$ maps bounded sets of $\mathbb{R}^{+} \times X_{\alpha}$ into bounded sets of $X$.

If $u_{0} \in X_{\alpha}$ with $u_{0} \geq \sigma e_{1}$ and $\alpha q<1 / 2$ for some $1 / 2<q<$ 1 , then the IVP(1) has at least one positive mild solution $u \in$ $C\left([0, T), X_{\alpha}\right)$. And if $T<\infty$, one has $\lim _{t \in T^{-}}\|u(t)\|_{\alpha}=\infty$.

Proof. For any $t_{0} \geq 0$ and $x_{0} \in X_{\alpha}$ with $x_{0} \geq \sigma e_{1}$, we first prove that the initial value problem (IVP) of fractional evolution equations

$$
\begin{gathered}
D_{t_{0}}^{q} u(t)+A u(t)=f(t, u(t)), \quad t>t_{0}, \\
u\left(t_{0}\right)=x_{0}
\end{gathered}
$$

has at least one positive mild solution on $J=\left[t_{0}, t_{0}+h_{t_{0}}\right]$, where $h_{t_{0}}$ is a positive constant and will be given later.

Let $R_{t_{0}}:=2 M\left(\left\|x_{0}\right\|_{\alpha}+1\right)+\sigma e_{1}>0$. Denote

$$
\Omega_{R_{t_{0}}}:=\left\{u \in C\left(J, X_{\alpha}\right):\|u(t)\|_{\alpha} \leq R_{t_{0}}, u(t) \geq \sigma e_{1}, t \in J\right\} .
$$

Then $\Omega_{R_{t_{0}}} \subset C\left(J, X_{\alpha}\right)$ is a nonempty bounded convex closed set. The assumption $\left(H_{2}\right)$ implies that there is a constant $C=$ $C\left(t_{0}\right)>0$ such that

$$
\|f(t, u)\| \leq C
$$

for any $t \in J$ and $u \in \Omega_{R_{t_{0}}}$. 
Define an operator $Q$ by

$$
\begin{aligned}
(Q u)(t)= & U\left(t-t_{0}\right) x_{0} \\
& +\int_{t_{0}}^{t}(t-s)^{q-1} V(t-s) f(s, u(s)) d s, \quad t \in J .
\end{aligned}
$$

By the continuity of $f$, it is not difficult to prove that $Q$ : $C\left(J, X_{\alpha}\right) \rightarrow C\left(J, X_{\alpha}\right)$ is continuous. By the positivity of the semigroup $S(t)(t \geq 0)$, the assumption $\left(H_{1}\right)$, and (20), we easily see that $(Q u)(t) \geq\left(Q \sigma e_{1}\right)(t)$. Clearly, the positive mild solution of the $\operatorname{IVP}(17)$ on $J$ is equivalent to the fixed point of operator $Q$ in $\Omega_{R_{t_{0}}}$. We will use Schauder fixed point theorem to prove that $Q$ has fixed points in $\Omega_{R_{t_{0}}}$.

We first prove that $Q: \Omega_{R_{t_{0}}} \rightarrow \Omega_{R_{t_{0}}}$ is continuous. Let $h_{t_{0}} \leq\left[M(1-\alpha) \Gamma(1+q(1-\alpha))\left(\left\|x_{0}\right\|_{\alpha}+1\right) / M_{\alpha} C \Gamma(2-\alpha)\right]^{1 / q(1-\alpha)}$. For any $u \in \Omega_{R_{t_{0}}}$ and $t \in J$, by Lemma 6, (10), (19), and (20), we have

$$
\begin{aligned}
& \|(Q u)(t)\|_{\alpha} \\
& \leq\left\|U\left(t-t_{0}\right) x_{0}\right\|_{\alpha}+\left\|\int_{t_{0}}^{t}(t-s)^{q-1} V(t-s) f(s, u(s)) d s\right\|_{\alpha} \\
& \leq M\left\|x_{0}\right\|_{\alpha}+\int_{t_{0}}^{t}(t-s)^{q-1}\left\|A^{\alpha} V(t-s)\right\| \cdot\|f(s, u(s))\| d s \\
& \leq M\left\|x_{0}\right\|_{\alpha}+q M_{\alpha} C \int_{t_{0}}^{t}(t-s)^{q(1-\alpha)-1} d s \cdot \int_{0}^{\infty} \theta^{1-\alpha} \eta_{q}(\theta) d \theta \\
& =M\left\|x_{0}\right\|_{\alpha}+\frac{M_{\alpha} C \Gamma(2-\alpha)}{(1-\alpha) \Gamma(1+q(1-\alpha))} h_{t_{0}}^{q(1-\alpha)} \leq R_{t_{0}} .
\end{aligned}
$$

Let $v_{0} \equiv \sigma e_{1}$. Then $v_{0}(t)=\sigma e_{1}$ for any $t \in J$ and

$$
\phi(t) \triangleq D_{t_{0}}^{q} v_{0}(t)+A v_{0}(t)=\lambda_{1} \sigma e_{1} \leq f\left(t, \sigma e_{1}\right), \quad t \in J .
$$

By the positivity of the semigroup $S(t)(t \geq 0)$, assumption $\left(H_{1}\right)$, and $(20)$, for any $t \in J$, we have

$$
\begin{aligned}
\sigma e_{1} & =v_{0}(t) \\
& =U\left(t-t_{0}\right) v_{0}\left(t_{0}\right)+\int_{t_{0}}^{t}(t-s)^{q-1} V(t-s) \phi(s) d s \\
& \leq U\left(t-t_{0}\right) x_{0}+\int_{t_{0}}^{t}(t-s)^{q-1} V(t-s) f\left(s, \sigma e_{1}\right) d s \\
& =\left(Q \sigma e_{1}\right)(t) \leq(Q u)(t) .
\end{aligned}
$$

Thus, $Q: \Omega_{R_{t_{0}}} \rightarrow \Omega_{R_{t_{0}}}$ is continuous.

By using a similar argument as in the proof of Theorem 3.1 in [14], we can prove that $Q: \Omega_{R_{t_{0}}} \rightarrow \Omega_{R_{t_{0}}}$ is a compact operator. Hence by Schauder fixed point theorem, the operator $Q$ has at least one fixed point $u^{*}$ in $\Omega_{R_{t_{0}}}$, which satisfies $u^{*}(t) \geq \sigma e_{1}>0$ for all $t \in J$. Hence $u^{*}$ is a positive mild solution of the IVP(1) on $J$.

Therefore, there exists $\left[0, h_{0}\right]$ such that the $\operatorname{IVP}(1)$ has at least one positive mild solution $u^{*} \in C\left(\left[0, h_{0}\right], X_{\alpha}\right)$. Now, by the standard proof method of extension theorem of initial value problem, $u^{*}$ can be extended to a saturated solution $u \in C\left([0, T), X_{\alpha}\right)$ of the $\operatorname{IVP}(1)$, whose existence interval is $[0, T)$, and if $T<\infty$, we have $\lim _{t \rightarrow T^{-}}\|u(t)\|_{\alpha}=\infty$.

For any $T>0$ and $r>0$, define $\Omega_{r}$ as in (15). If $f(t, u)$ is increasing in $\Omega_{r}$, that is, $f(t, u)$ satisfies the condition

$\left(H_{1}\right)^{*}$ for any $u_{1}, u_{2} \in \Omega_{r}$ with $u_{1}(t) \leq u_{2}(t)$ for all $t \in[0, T]$, we have

$$
f\left(t, u_{1}(t)\right) \leq f\left(t, u_{2}(t)\right), \quad t \in[0, T],
$$

then we have $f(t, u(t)) \geq f\left(t, \sigma e_{1}\right)$ for any $u \in \Omega_{r}$ and $t \in[0, T]$. On the other hand, if $f(t, u)$ satisfies linear growth condition, then it maps the bounded sets into the bounded sets. Hence by Theorem 12, we have the following existence result.

Corollary 13. Let $-A: D(A) \subset X \rightarrow X$ be the infinitesimal generator of a positive and compact analytic semigroup $S(t)(t \geq 0)$ of uniformly bounded linear operators. Assume that $f \in C\left(\mathbb{R}^{+} \times X_{\alpha}, X\right)$ satisfies condition $\left(H_{1}\right)^{*}$ and

$\left(\mathrm{H}_{2}\right)^{*}$ there exists a constant $a_{f}>0$ such that

$$
\|f(t, x)\| \leq a_{f}\left(1+\|x\|_{\alpha}\right)
$$

for all $t \in[0, T]$ and $x \in X_{\alpha}$.

If $f\left(t, \sigma e_{1}\right) \geq \lambda_{1} \sigma e_{1}$ for all $t \in[0, T], u_{0} \in X_{\alpha}$ with $u_{0} \geq$ $\sigma e_{1}$ and $\alpha q<1 / 2$ for some $1 / 2<q<1$, then the IVP(1) has at least one positive mild solution $u \in C\left([0, T), X_{\alpha}\right)$. And if $t<\infty$, one has $\lim _{t \rightarrow T^{-}}\|u(t)\|_{\alpha}=\infty$.

Since the analytic semigroup is norm continuous, it follows that we can delete the compactness condition on the analytic semigroup $S(t)(t \geq 0)$ and obtain the following existence result.

Theorem 14. Assume that $-A: D(A) \subset X \rightarrow X$ is the infinitesimal generator of a positive analytic semigroup $S(t)(t \geq 0)$ of uniformly bounded linear operators, and that $f \in C\left(\mathbb{R}^{+} \times\right.$ $\left.X_{\alpha}, X\right)$ satisfies the condition $\left(H_{1}\right)$ and

$\left(H_{3}\right)$ for any $T>0$ and $r>0, f\left(t, \Omega_{r}\right):=\left\{f(t, u): u \in \Omega_{r}\right\}$ is relatively compact in $X_{\alpha}$ for all $t \in[0, T]$, where $\Omega_{r}$ is defined as in (15).

If $u_{0} \in X_{\alpha}$ with $u_{0} \geq \sigma e_{1}$ and $\alpha q<1 / 2$ for some $1 / 2<q<$ 1 , then the IVP(1) has at least one positive mild solution $u \in$ $C\left([0, T), X_{\alpha}\right)$. And if $t<\infty$, one has $\lim _{t \rightarrow T^{-}}\|u(t)\|_{\alpha}=\infty$.

Proof. For any $t_{0} \geq 0$ and $x_{0} \in X_{\alpha}$ with $x_{0} \geq \sigma e_{1}$, we first prove that the $\operatorname{IVP}(17)$ has at least one positive mild solution on $J=\left[t_{0}, t_{0}+h_{t_{0}}\right]$, where $h_{t_{0}}>0$ is a constant and will be specified later. Define an operator $Q$ as in (20). Let $R_{t_{0}}=$ $2 M\left(\left\|x_{0}\right\|_{\alpha}+1\right)+\sigma e_{1}$. Write $\Omega_{R_{t_{0}}}$ as in (18). The condition $\left(H_{3}\right)$ 
implies that $f\left(t, \Omega_{R_{t_{0}}}\right)$ is bounded for any $t \in J$, that is, there is a positive constant $C=C\left(t_{0}\right)$ such that

$$
\|f(t, u(t))\| \leq C, \quad t \in J, u \in \Omega_{R_{t_{0}}} .
$$

Let $h_{t_{0}} \leq\left[M(1-\alpha) \Gamma(1+q(1-\alpha))\left(\left\|x_{0}\right\|_{\alpha}+1\right) / M_{\alpha} C \Gamma(2-\right.$ $\alpha)]^{1 / q(1-\alpha)}$. A similar argument as in the proof of Theorem 12 shows that $Q: \Omega_{R_{t_{0}}} \rightarrow \Omega_{R_{t_{0}}}$ is continuous and $Q \Omega_{R_{t_{0}}}$ is equicontinuous.

Thus, for any $D \subset \Omega_{R_{t_{0}}}$, let $D(t):=\{u(t): u \in D\}, t \in J$. Since $Q D \subset Q \Omega_{R_{t_{0}}} \subset \Omega_{R_{t_{0}}}$ is equicontinuous and bounded, by Lemma 8 , we have

$$
\beta(Q D)=\max _{t \in J} \beta((Q D)(t)) .
$$

Now, let $D=\left\{u_{m}: m=1,2, \ldots\right\} \subset \Omega_{R_{t_{0}}}$ with $D \subset$ $\overline{\mathrm{Co}}\left(\left\{y_{0}\right\} \cup \mathrm{QD}\right)$ for some $y_{0} \in \Omega_{R_{t_{0}}}$. It is obvious that

$$
\begin{aligned}
& \left\|(t-s)^{q-1} V(t-s) f(s, u(s))\right\|_{\alpha} \\
& \quad \leq \frac{C M_{\alpha} q \Gamma(2-\alpha)}{\Gamma(1+q(1-\alpha))}(t-s)^{q-1} \in L\left(J, \mathbb{R}^{+}\right) .
\end{aligned}
$$

Hence by Lemma 9 and (20), we have

$$
\begin{aligned}
\beta & ((Q D)(t)) \\
& =\beta\left(U\left(t-t_{0}\right) x_{0}+\int_{t_{0}}^{t}(t-s)^{q-1} V(t-s) f(s, D(s)) d s\right) \\
& =\beta\left(\int_{t_{0}}^{t}(t-s)^{q-1} V(t-s) f(s, D(s)) d s\right) \\
& \leq 2 \int_{t_{0}}^{t}(t-s)^{q-1}\left\|A^{\alpha} V(t-s)\right\| \cdot \beta(f(s, D(s))) d s \\
& \leq \frac{2 M_{\alpha} q \Gamma(2-\alpha)}{\Gamma(1+q(1-\alpha))} \int_{t_{0}}^{t}(t-s)^{q(1-\alpha)-1} \cdot \beta(f(s, D(s))) d s \\
& =0 .
\end{aligned}
$$

It follows that $\beta((Q D)(t))=0$ for all $t \in J$. By Lemma 8 and (27), we have $\beta(Q D)=\max _{t \in J} \beta((Q D)(t))=0$. Thus, we have

$$
\begin{aligned}
\beta(D) & \leq \beta\left(\overline{\mathrm{Co}}\left(\left\{y_{0}\right\} \cup Q D\right)\right) \\
& =\beta\left(\left\{y_{0}\right\} \cup Q D\right)=\beta(Q D)=0 .
\end{aligned}
$$

This implies that $D$ is relatively compact. Therefore, by Mönch fixed point theorem, the operator $Q$ has at least one fixed point $u^{*} \in \Omega_{R_{t_{0}}}$, which satisfies $u^{*}(t) \geq \sigma e_{1}>0$ for all $t \in J$. Hence $u^{*}$ is a positive mild solution of the $\operatorname{IVP}(17)$ on $J$.

Therefore, there exists $\left[0, h_{0}\right]$ such that the $\operatorname{IVP}(1)$ has at least one positive mild solution $u^{*} \in C\left(\left[0, h_{0}\right], X_{\alpha}\right) . u^{*}$ can be extended to a saturated solution $u \in C\left([0, T), X_{\alpha}\right)$ of $\operatorname{IVP}(1)$, whose existence interval is $[0, T)$ and when $t \leq \infty$, we have $\lim _{t \rightarrow T^{-}}\|u(t)\|_{\alpha}=\infty$.

\section{Positive Mild Solutions of Parabolic Equations}

Let $\Omega \subset \mathbb{R}^{N}$ be a bounded domain with a sufficiently smooth boundary $\partial \Omega$. Let

$$
A(x, D) u=-\sum_{i, j=1}^{N} \frac{\partial}{\partial x_{i}}\left(a_{i j}(x) \frac{\partial u}{\partial x_{j}}\right)+a_{0}(x) u
$$

be a uniformly elliptic differential operator of divergence form in $\bar{\Omega}$, where the coefficients $a_{i j} \in C^{1+\mu}(\bar{\Omega})(i, j=$ $1,2, \ldots, N)$ and $a_{0} \in C^{\mu}(\bar{\Omega})$ for some $\mu \in(0,1)$. We assume that $\left[a_{i j}(x)\right]_{N \times N}$ is a positive define symmetric matric for every $x \in \bar{\Omega}$, and there exists a constant $v>0$ such that

$$
\begin{array}{r}
\sum_{i, j=1}^{N} a_{i j}(x) \xi_{i} \xi_{j} \geq v|\xi|^{2} \\
\forall \xi=\left(\xi_{1}, \xi_{2}, \ldots, \xi_{N}\right) \in \mathbb{R}^{N}, x \in \bar{\Omega} .
\end{array}
$$

Let $a_{0}(x) \geq 0$ on $\bar{\Omega}$. We use $(x, t, \eta)$ to denote a generic point of $\bar{\Omega} \times \mathbb{R}^{+} \times \mathbb{R}$, where $\mathbb{R}=[0,+\infty)$ and $\mathbb{R}=$ $(-\infty,+\infty)$. Let $F: \bar{\Omega} \times \mathbb{R}^{+} \times \mathbb{R} \rightarrow \mathbb{R}$ be a continuous function. We discuss the existence of positive mild solutions for the parabolic initial boundary value problem (IBVP)

$$
\begin{gathered}
\frac{\partial^{q}}{\partial t^{q}} u(x, t)+A(x, D) u(x, t)=F(x, t, u(x, t)) \quad \text { in } \Omega \times \mathbb{R}^{+}, \\
\left.u\right|_{\partial \Omega}=0, \\
u(x, 0)=\varphi(x) \quad \text { in } \Omega,
\end{gathered}
$$

where $0<q<1$ is a constant.

Let $\lambda_{1}$ be the smallest positive real eigenvalue of elliptic operator $A(x, D)$ under the Dirichlet boundary condition $\left.u\right|_{\partial \Omega}=0$. It is well known (cf. Amann $[22,28]$ ) that $\lambda_{1}>0$. Let $e_{1}(x)$ be the positive eigenvector corresponding to $\lambda_{1}$. Assume that $F: \bar{\Omega} \times \mathbb{R}^{+} \times \mathbb{R} \rightarrow \mathbb{R}$ is continuous and satisfies the following conditions.

$\left(F_{1}\right)$ For any $T>0$ and $r>0$, there exists a constant $\sigma>0$ such that

$F(x, t, \eta) \geq F\left(x, t, \sigma e_{1}(x)\right) \geq \lambda_{1} \sigma e_{1}(x), \quad t \in[0, T], x \in \bar{\Omega}$,

where $\eta \in \mathbb{R}$ with $\sigma e_{1}(x) \leq \eta \leq r$.

$\left(F_{2}\right)$ For any $T>0$, there exists a constant $a_{f}>0$ such that

$$
|F(x, t, \eta)| \leq a_{f}(1+|\eta|), \quad x \in \bar{\Omega}, t \in[0, T] .
$$
by

Let $X=L^{2}(\Omega)$. Define an operator $A: D(A) \subset X \rightarrow X$

$$
D(A)=H^{2}(\Omega) \cap H_{0}^{1}(\Omega), \quad A u=A(x, D) u .
$$


It is well known (cf. Li [29]) that $-A$ generates a compact analytic semigroup $S(t)(t \geq 0)$ and $D\left(A^{1 / 2}\right)=H_{0}^{1}(\Omega)$. By the maximum principle of the equation of the parabolic type, it is easy to prove that $S(t)(t \geq 0)$ is also a positive semigroup in $X$. The assumptions $\left(F_{1}\right)$ and $\left(F_{2}\right)$ imply that the mapping $f: \mathbb{R}^{+} \times X_{1 / 2} \rightarrow X$ defined by

$$
f(t, u)(\cdot)=F(\cdot, t, u(\cdot)), \quad t \in \mathbb{R}^{+}, u \in H_{0}^{1}(\Omega),
$$

is continuous and satisfies the conditions $\left(H_{1}\right)$ and $\left(H_{2}\right)$. Thus, the IBVP(33) can be rewritten into the abstract form of IVP(1). By Theorem 12, we have the following existence result for the $\operatorname{IBVP}(33)$.

Theorem 15. Assume that $F: \bar{\Omega} \times \mathbb{R}^{+} \times \mathbb{R} \rightarrow \mathbb{R}$ is continuous and satisfies conditions $\left(F_{1}\right)$ and $\left(F_{2}\right)$. If $\varphi \in H_{0}^{1}(\Omega)$ with $\varphi(x) \geq \sigma e_{1}(x)$ for any $x \in \bar{\Omega}$ and $1 / 2<q<1$, then the $\operatorname{IBVP}(33)$ has at least one positive mild solution $u$ that satisfies $u(x, t) \geq \sigma e_{1}(x)$ for any $x \in \bar{\Omega}$ and $t \in[0, T]$. And if $T<+\infty$, one has $\lim _{t \rightarrow T^{-}}|u(t)|=+\infty$.

\section{Acknowledgments}

This research was supported by the NNSF of China (Grant no. 11261053), the Fundamental Research Funds for the Gansu Universities, and the Project of NWNU-LKQN-11-3.

\section{References}

[1] K. S. Miller and B. Ross, An Introduction to the Fractional Calculus and Fractional Differential Equations, A Wiley-Interscience Publication, John Wiley \& Sons, New York, NY, USA, 1993.

[2] I. Podlubny, Fractional Differential Equations, vol. 198 of Mathematics in Science and Engineering, Academic Press, San Diego, Calif, USA, 1999.

[3] A. A. Kilbas, H. M. Srivastava, and J. J. Trujillo, Theory and Applications of Fractional Differential Equations, vol. 204 of North-Holland Mathematics Studies, Elsevier Science B.V., Amsterdam, The Netherlands, 2006.

[4] V. Lakshmikantham, S. Leela, and J. Devi, Theory of Fractional Dynamic Systems, Cambridge Scientific Publishers, Cambridge, UK, 2009.

[5] K. Diethelm, The Analysis of Fractional Differential Equations, vol. 2004 of Lecture Notes in Mathematics, Springer, Berlin, Germany, 2010.

[6] R. P. Agarwal, M. Belmekki, and M. Benchohra, "A survey on semilinear differential equations and inclusions involving Riemann-Liouville fractional derivative," Advances in Difference Equations, vol. 2009, Article ID 981728, 47 pages, 2009.

[7] R. P. Agarwal, M. Benchohra, and S. Hamani, "A survey on existence results for boundary value problems of nonlinear fractional differential equations and inclusions," Acta Applicandae Mathematicae, vol. 109, no. 3, pp. 973-1033, 2010.

[8] R. P. Agarwal, V. Lakshmikantham, and J. J. Nieto, "On the concept of solution for fractional differential equations with uncertainty," Nonlinear Analysis. Theory, Methods \& Applications, vol. 72, no. 6, pp. 2859-2862, 2010.

[9] M. M. El-Borai, "Some probability densities and fundamental solutions of fractional evolution equations," Chaos, Solitons and Fractals, vol. 14, no. 3, pp. 433-440, 2002.
[10] M. M. El-Borai, “The fundamental solutions for fractional evolution equations of parabolic type," Journal of Applied Mathematics and Stochastic Analysis, no. 3, pp. 197-211, 2004.

[11] M. M. El-Borai, "Semigroups and some nonlinear fractional differential equations," Applied Mathematics and Computation, vol. 149, no. 3, pp. 823-831, 2004.

[12] M. El-Borai, K. El-Nadi, and E. El-Akabawy, "Fractional evolution equations with nonlocal conditions," International Journal of Applied Mathematics and Mechanics, vol. 4, no. 6, pp. 1-12, 2008.

[13] Y. Zhou and F. Jiao, "Nonlocal Cauchy problem for fractional evolution equations," Nonlinear Analysis. Real World Applications, vol. 11, no. 5, pp. 4465-4475, 2010.

[14] J. Wang and Y. Zhou, "A class of fractional evolution equations and optimal controls," Nonlinear Analysis. Real World Applications, vol. 12, no. 1, pp. 262-272, 2011.

[15] Y. Zhou and F. Jiao, "Existence of mild solutions for fractional neutral evolution equations," Computers \& Mathematics with Applications, vol. 59, no. 3, pp. 1063-1077, 2010.

[16] J. Wang, Y. Zhou, and W. Wei, "A class of fractional delay nonlinear integrodifferential controlled systems in Banach spaces," Communications in Nonlinear Science and Numerical Simulation, vol. 16, no. 10, pp. 4049-4059, 2011.

[17] Z. Tai and X. Wang, "Controllability of fractional-order impulsive neutral functional infinite delay integrodifferential systems in Banach spaces," Applied Mathematics Letters, vol. 22, no. 11, pp. 1760-1765, 2009.

[18] A. Debbouche and D. Baleanu, "Controllability of fractional evolution nonlocal impulsive quasilinear delay integrodifferential systems," Computers \& Mathematics with Applications, vol. 62, no. 3, pp. 1442-1450, 2011.

[19] G. M. Mophou, "Existence and uniqueness of mild solutions to impulsive fractional differential equations," Nonlinear Analysis. Theory, Methods \& Applications, vol. 72, no. 3-4, pp. 1604-1615, 2010.

[20] R.-N. Wang, T.-J. Xiao, and J. Liang, "A note on the fractional Cauchy problems with nonlocal initial conditions," Applied Mathematics Letters, vol. 24, no. 8, pp. 1435-1442, 2011.

[21] P. Sobolevskii, "Equations of parabolic type in a Banach space," American Mathematics Society Translations. Series 2, vol. 49, pp. $1-62,1966$.

[22] H. Amann, "Periodic solutions of semilinear parabolic equations," in Nonlinear Analysis, pp. 1-29, Academic Press, New York, NY, USA, 1978.

[23] A. Pazy, Semigroups of Linear Operators and Applications to Partial Differential Equations, vol. 44 of Applied Mathematical Sciences, Springer, New York, NY, USA, 1983.

[24] H. Liu and J.-C. Chang, "Existence for a class of partial differential equations with nonlocal conditions," Nonlinear Analysis. Theory, Methods \& Applications, vol. 70, no. 9, pp. 3076-3083, 2009.

[25] K. Deimling, Nonlinear Functional Analysis, Springer, Berlin, Germany, 1985.

[26] D. Guo, V. Lakshmikantham, and X. Liu, Nonlinear Integral Equations in Abstract Spaces, vol. 373 of Mathematics and Its Applications, Kluwer Academic Publishers, Dordrecht, The Netherlands, 1996.

[27] J. Liang and T.-J. Xiao, "Solvability of the Cauchy problem for infinite delay equations," Nonlinear Analysis. Theory, Methods \& Applications, vol. 58, no. 3-4, pp. 271-297, 2004. 
[28] H. Amann, "Nonlinear operators in ordered Banach spaces and some applications to nonlinear boundary value problems," in Nonlinear Operators and the Calculus of Variations, vol. 543 of Lecture Notes in Mathematics, pp. 1-55, Springer, Berlin, Germany, 1976.

[29] Y. Li, "Existence and asymptotic stability of periodic solution for evolution equations with delays," Journal of Functional Analysis, vol. 261, no. 5, pp. 1309-1324, 2011. 


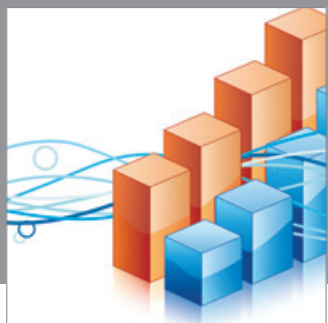

Advances in

Operations Research

mansans

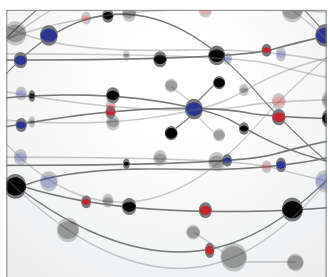

The Scientific World Journal
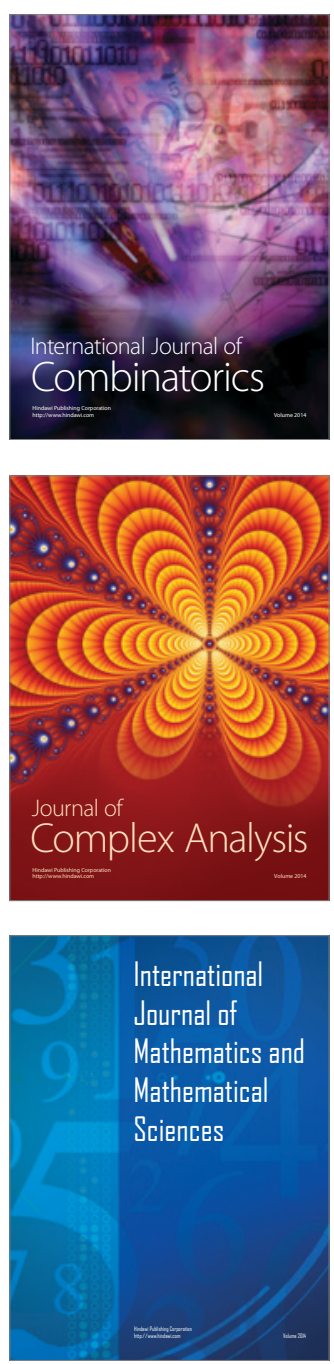
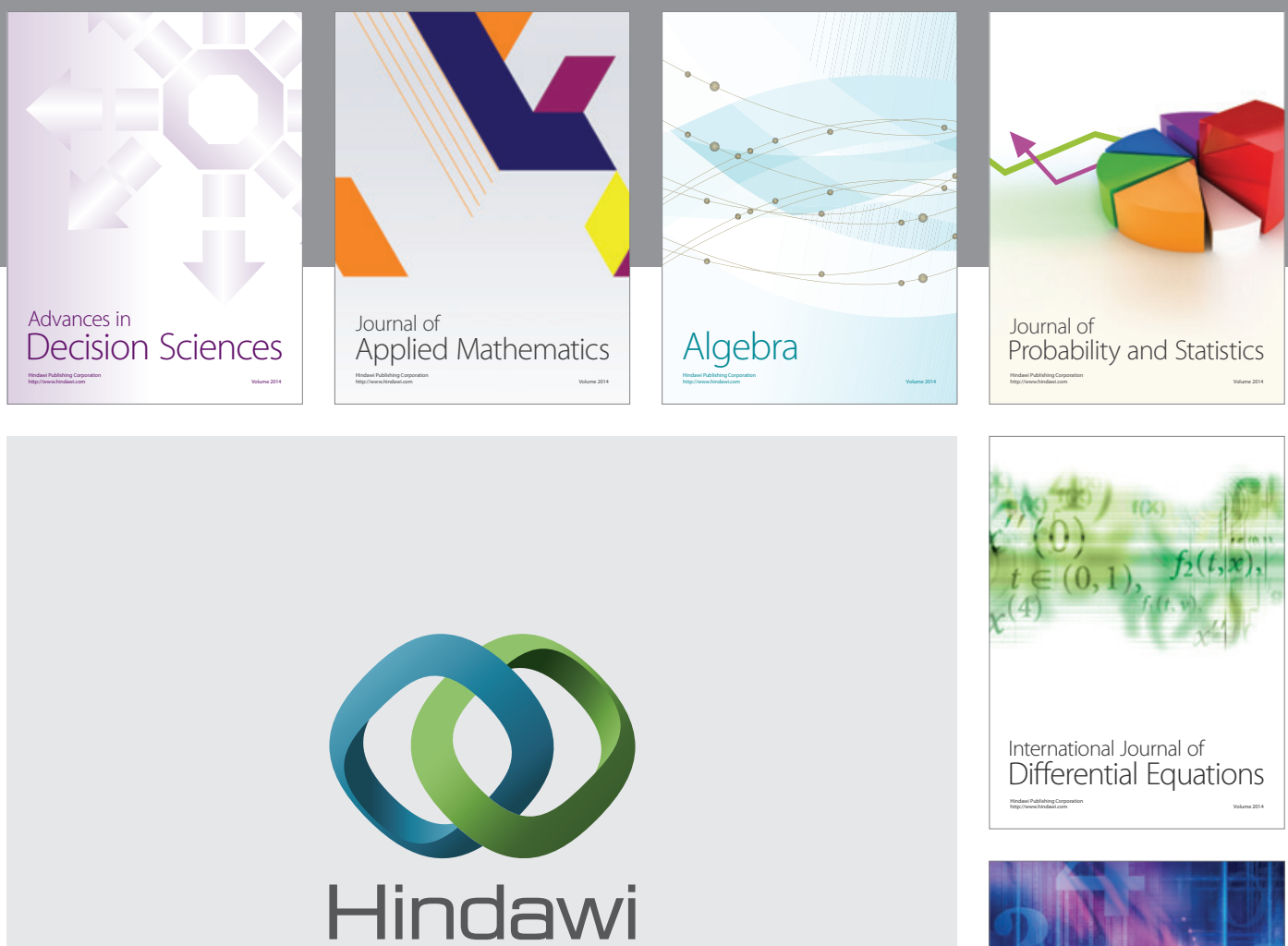

Submit your manuscripts at http://www.hindawi.com
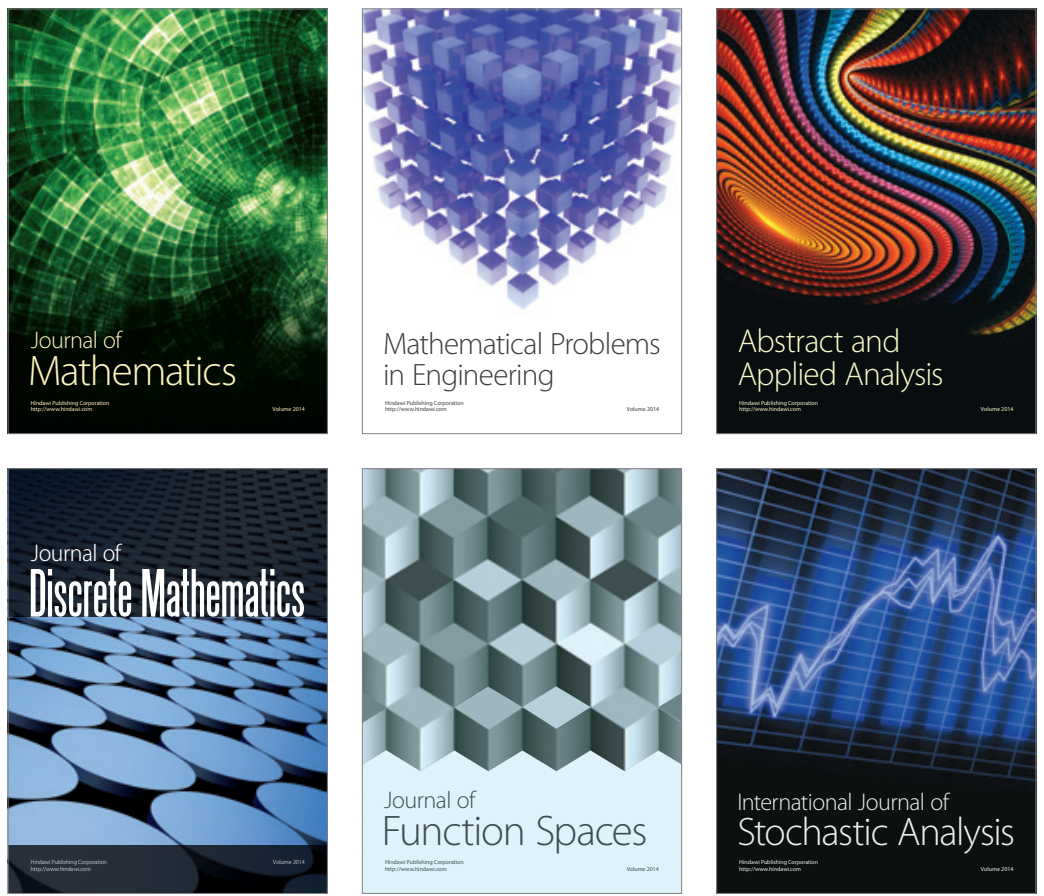

Journal of

Function Spaces

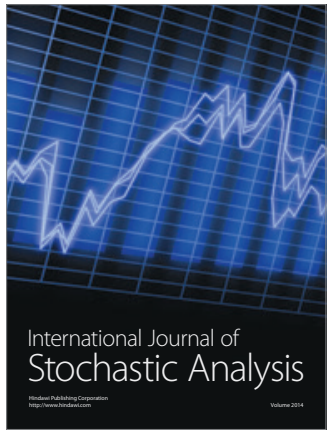

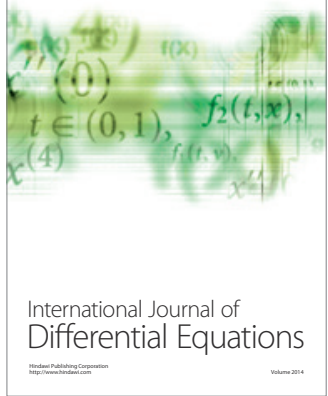
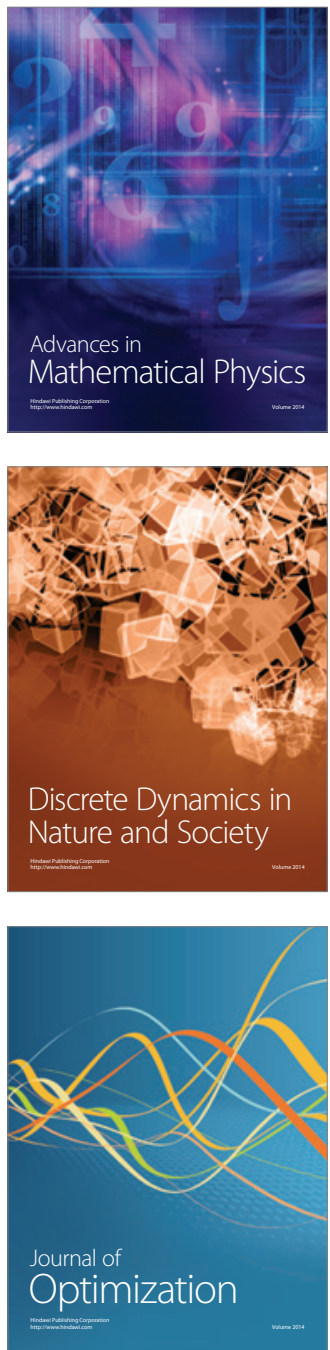